\title{
Local Existence Theorem of Fractional Differential Equations in $\mathrm{L}^{\mathrm{p}}$ Space Joseph Gh. Abdulahad \\ Faculty of Science University of Zakho \\ Shayma A. Murad \\ Faculty of Science \\ University of Duhok
}

\section{Received on: 18/04/2011}

Accepted on: 02/11/2011

\begin{abstract}
We proved the existence of P-integrable solution in $L^{p}(a, b)$-space, where $1 \leq p<\infty$ for the fractional differential equation which has the form:

${ }^{c} D^{\alpha} y(t)=f(t, y(t)) \quad 0<\alpha<1$

with boundary condition

$\gamma y(a)+\mu y(b)=c$

where $D^{\alpha}$ is the Caputo fractional derivative, $\gamma, \mu$ and $\mathrm{c}$ are positive constants with $\gamma+\mu \neq 0$. The contraction mapping principle has been used to establish our main result.
\end{abstract}

Keywords: Fractional differential equation, Caputo fractional derivative, $\mathrm{L}^{\mathrm{p}}$ space.

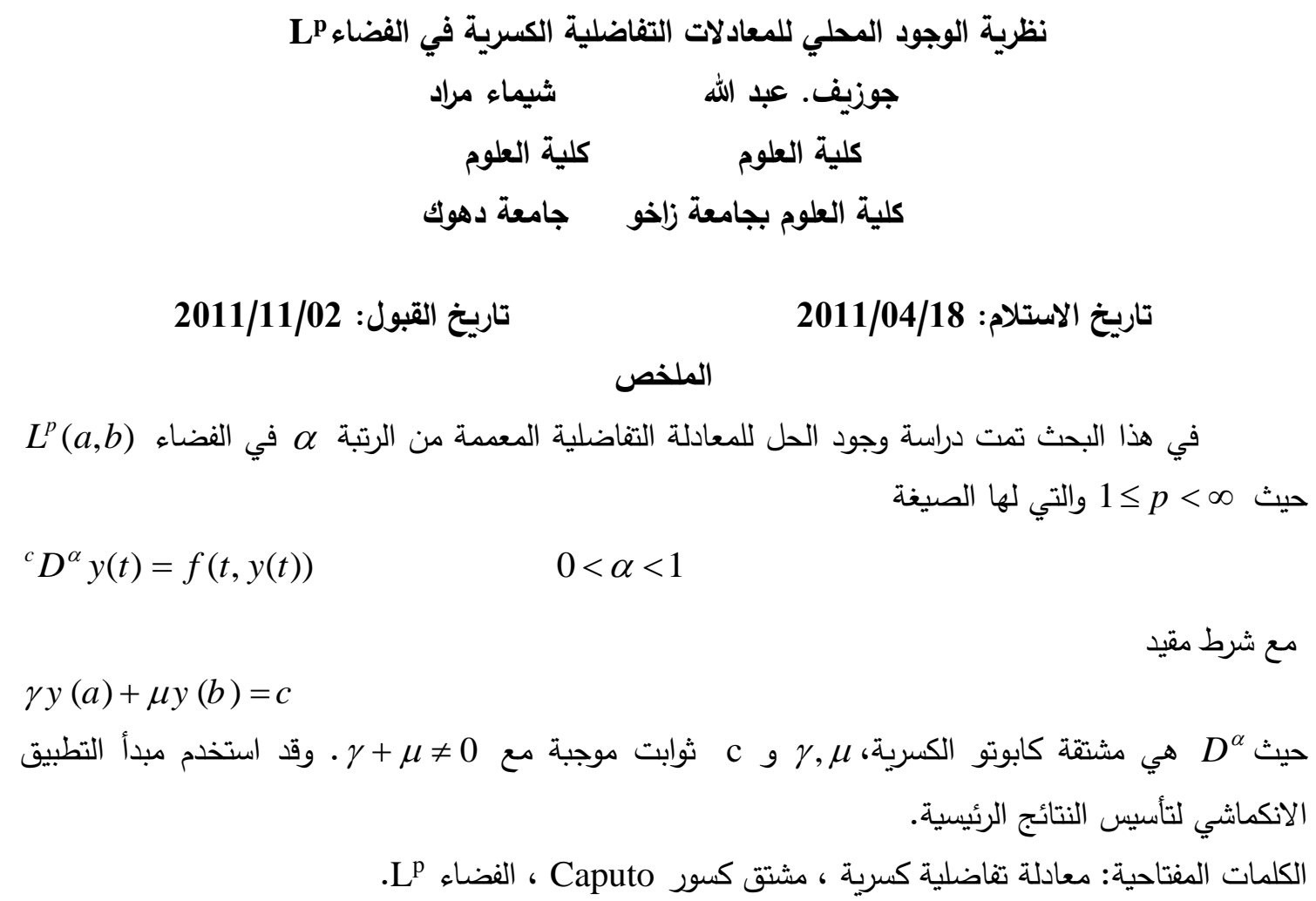

\section{Introduction}

Fractional differential equations have gained importance and popularity during the past three decades or so, due to mainly its demonstrated applications in numerous seemingly diverse fields of science and engineering. Fractional derivatives provide an excellent

instrument for the description of memory and hereditary properties of various materials and processes. This is the main advantage of fractional derivatives in 
comparison with classical integer-order models, in which such effects are in fact neglected. The advantages of fractional derivatives become apparent in modeling mechanical and electrical properties of real materials, and in many other fields see ([6],[7]).

Arora and Alshamani [1] proved a global existence and uniqueness theorem for the equation

$D^{\alpha} y(t)=f(t, y(t))$

to the case when the order $\alpha$ in (1.1) is taken to be $n-1<\alpha<n, \quad n \in Z^{+}, n \geq 2$ and satisfies the initial condition

$y^{(\alpha-i)}(a)=c_{i}$

where $a, c_{i} \in R, i=1,2, \ldots, n, c_{n}=0, f(t, y)$ is Lebsegue integrable function which satisfies the global Lipschitz condition. Hadid [5] studied local and global existence theorems of the nonlinear differential equation

$D^{\alpha} y(t)=f(t, y(t)) \quad 0<\alpha<1$

satisfying initial condition

$y\left(t_{0}\right)=t_{0}$

by Shauder and Tyconove fixed point theorems. Momani [8] studied local and global uniqueness theorems of the fractional differential equation (1.3) with the condition (1.4), by using Biharie's and Gronwell's inequalities. Benchohra et al [4] studied the existence of solutions for boundary value problems, for fractional differential equation(1.3), for each $t \in J=[0, T], \quad 0<\alpha<1$ and satisfying the boundary condition $\gamma y(0)+\mu y(T)=c$

By using Schaefer's fixed point theorem.

In this paper we impose some conditions on the existence of the solution of fractional differential equation (1.3), with boundary condition

$\gamma y(a)+\mu y(b)=c$

to be in the space of $L^{p}(a, b)$, Our method in this paper is by using the contraction mapping principle.

\section{Preliminaries}

In this section,we shall give a collection of definitions and lemmas which are needed in various places in this work.

\section{Definition 2.1 [2]} we define

Let $\mathrm{f}$ be a function which is defined almost everywhere (a.e) on [a,b]. for $\alpha>0$, $D_{a}^{-\alpha} f=\frac{1}{\Gamma(\alpha)} \int_{a}^{b}(b-t)^{\alpha-1} f(t) d t$

Provided that this integral (lebsegue) exists, where $\Gamma$ is gamma function.

\section{Definition 2.2 [6]}

For a function $\mathrm{f}$ defined on the interval $[\mathrm{a}, \mathrm{b}]$, the $\alpha$ th Caputo fractional derivative of $f$ is defined by

$\left(D_{a+}^{\alpha} f\right)(t)=\frac{1}{\Gamma(n-\alpha)} \int_{a}^{t}(t-s)^{n-\alpha-1} f^{(n)}(s, y(s)) d s$ 
where $n=[\alpha]+1$ and $[\alpha]$ denotes the integer part of $\alpha$.

Lemma 2.1 [2] If $\alpha>0$ and $f(t)$ is continuous on $[a, b]$, then $D^{-\alpha} f$ exists and it is continuous with respect to $t$ on $[a, b]$.

Lemma 2.2 [7] If $\alpha>0$ and $\mathrm{f}(\mathrm{t})$ belongs to $L(a, b)$, then ${ }_{a}^{t} D^{-\alpha} f$ exists for all $t \in[a, b]$ if $\alpha \geq 1$ and a.e. if $\alpha<1$.

Lemma 2.3 [3] If $\alpha, \beta>0$ and $f(t) \in L(a, b)$, then on $a \leq t \leq b$, we have:

a. $\int_{a}^{t}{ }_{a}^{s} D^{-\alpha} f d s={ }_{a}^{t} D^{-(\alpha+1)} f \quad$ or ${ }_{a}^{t} D^{-1}{ }_{a}^{s} D^{-\alpha} f={ }_{a}^{t} D^{-(\alpha+1)} f$

b. If $\alpha \geq 1$, then ${ }_{a}^{t} D^{-\alpha} f$ is absolutely continuous in $t \in[a, b]$.

c. $\frac{d}{d t}{ }_{a}^{t} D^{-(\alpha+1)} f={ }_{a}^{t} D^{-\alpha} f$, everywhere if $\alpha \geq 1$ and a.e. if $\alpha<1$.

d. ${ }_{a}^{t} D^{-(\alpha+\beta)} f={ }_{a}^{t} D^{-\alpha}{ }_{a}^{s} D^{-\beta} f$ a.e. if $\alpha+\beta \leq 1$.

Lemma 2.4 [9] (Hölder's inequality)

Let $X$ be a measurable space, let $p$ and $q$ satisfy $1<\mathrm{p}<\infty, 1<\mathrm{q}<\infty$, and $\frac{1}{p}+\frac{1}{q}=1$. If $\mathrm{f} \in \mathrm{L}^{\mathrm{p}}(\mathrm{X})$ and $\mathrm{g} \in \mathrm{L}^{\mathrm{q}}(\mathrm{X})$, then $(f g)$ belongs to $L(X)$ and satisfies

$$
\int_{X}|f g| d x \leq\left[\int_{X}|f|^{p} d x\right]^{\frac{1}{p}}\left[\int_{X}|g|^{q} d x\right]^{\frac{1}{q}}
$$

\section{Local Existence of P-integrable solution in $L^{p}(a, b)$ space}

In this section we prove some existence and uniqueness theorems in $L^{p}(a, b)$ space for the boundary value problems(1.3), with condition (1.5).

\section{Lemma 3.1 [4]}

Let $0<\alpha<1$ and $\mathrm{f}$ be continuous function. A function $\mathrm{y}$ is defined as

$$
y(t)=\frac{1}{\Gamma(\alpha)} \int_{a}^{t}(t-s)^{\alpha-1} f(s, y(s)) d s-\frac{\mu}{(\gamma+\mu) \Gamma(\alpha)} \int_{a}^{b}(b-s)^{\alpha-1} f(s, y(s)) d s+\frac{c}{\gamma+\mu}
$$

if and only if $\mathrm{y}$ is a solution of the fractional boundary value problem (1.3), (1.5).

For proof see [4].

The mean result is given in the following theorem

\section{Theorem 3.2}

Let the right hand side $f(t, y(t))$ of the fractional differential equation (1.3) satisfy the Lipschitz condition in $y$ with Lipschitz constant $\mathbf{M}$, that is, $\left|f\left(t, y_{1}\right)-f\left(t, y_{2}\right)\right| \leq M\left|y_{1}-y_{2}\right|$ on the domain $D$, where:

$$
D=\left\{(t, y):\left|t-t_{0}\right| \leq a,\left|y-y_{0}\right| \leq b\right\}
$$

and it is P-intgerable on $(\mathrm{a}, \mathrm{b})$. There exists a P-intgerable solution for the fractional differential equation (1.3) with the boundary condition (1.5). 
Proof. Let the mapping $T$ on $\mathrm{L}^{\mathrm{p}}(\mathrm{a}, \mathrm{b})$ be defined as:

$\operatorname{Tg}(t)=\frac{1}{\Gamma(\alpha)} \int_{a}^{t}(t-s)^{\alpha-1} f(s, g(s)) d s-\frac{\mu}{(\gamma+\mu) \Gamma(\alpha)} \int_{a}^{b}(b-s)^{\alpha-1} f(s, g(s)) d s+\frac{c}{\gamma+\mu}$

we have to prove that $T$ maps every function $g \in \mathrm{L}^{\mathrm{p}}(\mathrm{a}, \mathrm{b})$ into a function which belongs to $\mathrm{L}^{\mathrm{p}}(\mathrm{a}, \mathrm{b})$. Let:

$$
h(t)=\frac{1}{\Gamma(\alpha)} \int_{a}^{t}(t-s)^{\alpha-1} f(s, g(s)) d s-\frac{\mu}{(\gamma+\mu) \Gamma(\alpha)} \int_{a}^{b}(b-s)^{\alpha-1} f(s, g(s)) d s+\frac{c}{\gamma+\mu}
$$

by the lemma 2.1, the first and the second terms in the right hand side equation (3.3) is continuous for all $t \in[a, b]$, and for all $\alpha>0$. Hence $h(t)$ is continuous on $[a, b]$ and it is measurable .

$$
|h(t)|^{p}=\left|\frac{1}{\Gamma(\alpha) a} \int_{(t-s)}^{t}{ }^{\alpha-1} f(s, g(s)) d s-\frac{\mu}{(\gamma+\mu) \Gamma(\alpha) a}^{b}(b-s){ }^{\alpha-1} f(s, g(s)) d s+\frac{c}{\gamma+\mu}\right|
$$

We have show that $|h(t)|^{p}$ is Lebsegue integrable. Then, since $|f+g|^{p} \leq 2^{p}\left(|f|^{p}+|g|^{p}\right)$ and from equation (3.4), we have

$$
|h(t)|^{p} \leq 2^{p}\left|\frac{1}{\Gamma(\alpha)} \int_{a}^{t}(t-s)^{\alpha-1} f(s, g(s)) d s\right|^{p}+2^{p}\left|\frac{\mu}{(\gamma+\mu) \Gamma(\alpha) a} \int_{(b-s)}^{b-1} f(s, g(s)) d s-\frac{c}{\gamma+\mu}\right|^{p}
$$

the second term $\left|\frac{\mu}{(\gamma+\mu) \Gamma(\alpha) a} \int^{b}(b-s)^{\alpha-1} f(s, g(s)) d s-\frac{c}{\gamma+\mu}\right|^{p}$ is Lebsegue integrable.

Let $\quad(z(t))^{p}=\left[\frac{1}{\Gamma(\alpha)} \int_{a}^{t}(t-s)^{\alpha-1} f(s, g(s)) d s\right]^{p}$

Now we have to show that $(z(t))^{p}$ is Lebesgue integrable. Since $f(t, y(t)) \in L^{p}(a, b)$, and $(t-s)^{\alpha-1} \in L^{p}(a, b)$, then by HÖlder inequality $\left(\frac{1}{q}+\frac{1}{p}=1\right)$ and from equation (3.5), we obtain:

$$
\begin{aligned}
& (z(t))^{p} \leq\left[\left(\frac{1}{\Gamma(\alpha)} \int_{a}^{t}(t-s)^{q(\alpha-1)} d s\right)^{\frac{1}{q}}\left(\int_{a}^{t} f^{p}(s, y(s)) d s\right)^{\frac{1}{p}}\right]^{p} \\
& (z(t))^{p} \leq\left(\frac{1}{\Gamma(\alpha)} \int_{a}^{t}(t-s)^{q(\alpha-1)} d s\right)^{\frac{p}{q}} \int_{a}^{t} f^{p}(s, y(s)) d s \\
& (z(t))^{p} \leq \frac{(t-a)^{p \alpha-1}}{\left(\frac{p \alpha-1}{p-1}\right)^{p-1}} \int_{a}^{p}(s, y(s)) d s
\end{aligned}
$$

by Lemma 2.3.a, for $a \leq t \leq b$ we have 


$$
\begin{aligned}
& \int_{a}^{t}\left(\int_{a}^{s} f^{p}(\tau, y(\tau)) d \tau\right) d s=\int_{a}^{t}\left(\int_{a}^{s}(s-\tau)^{1-1} f^{p}(\tau, y(\tau)) d \tau\right) d s \\
& =\int_{a}^{t}\left({ }_{a}^{t} D^{-1} f^{p}(s, y(s))\right) d s={ }_{a}^{t} D^{-2} f^{p}
\end{aligned}
$$

From Definition 2.1, equation (3.8)becomes

$$
\int_{a}^{t}\left(\int_{a}^{s} f^{p}(\tau, y(\tau)) d \tau\right) d s=\frac{1}{\Gamma(2)} \int_{a}^{t}(t-s) f^{p}(s, y(s)) d s
$$

Thus by Lemma 2.2, it follows that $\int_{a}^{t} f^{p}$ is Lebesgue integrable for all $t \in[a, b]$. If there exist a Lebesgue integrable function $\mathrm{w}(\mathrm{t})$ on $[\mathrm{a}, \mathrm{b}]$ such that $|f(t)| \leq w(t)$ a.e on $[a, b]$, where $f(t)$ is measurable then $f(t)$ is Lebesgue integrable function. Hence $(z(t))^{p}$ is Lebesgue integrable. Thus $|h(t)|^{p}$ Lebesgue integrable and therefore $\mathrm{T}$ maps $\mathrm{L}^{\mathrm{p}}(\mathrm{a}, \mathrm{b})$ into itself. To show that $\mathrm{T}$ is a contraction mapping, Let $g_{1}, g_{1} \in L^{p}(a, b)$

$$
\begin{aligned}
&\left\|T\left(g_{1}(t)\right)-T\left(g_{2}(t)\right)\right\|_{p}^{p}=\| \frac{1}{\Gamma(\alpha)} \int_{a}^{t}(t-s)^{\alpha-1}\left(f\left(s, g_{1}(s)\right)-f\left(s, g_{2}(s)\right)\right) d s \\
&-\frac{\mu}{(\gamma+\mu) \Gamma(\alpha)} \int_{a}^{b}(b-s)^{\alpha-1}\left(f\left(s, g_{1}(s)\right)-f\left(s, g_{2}(s)\right)\right) d s \|_{p}^{p} \\
&=\int_{a}^{b}\left|\frac{1}{\Gamma(\alpha)} \int_{a}^{t}(t-s)^{\alpha-1}\left(f\left(s, g_{1}(s)\right)-f\left(s, g_{2}(s)\right)\right) d s-\frac{\mu}{(\gamma+\mu) \Gamma(\alpha)} \int_{a}^{b}(b-s)^{\alpha-1}\left(f\left(s, g_{1}(s)\right)-f\left(s, g_{2}(s)\right)\right) d s\right| d t
\end{aligned}
$$$$
\leq \frac{2^{p}}{(\Gamma(\alpha))^{p}} \int_{a}^{b}\left(\int_{a}^{t}(t-s)^{\alpha-1}\left|f\left(s, g_{1}(s)\right)-f\left(s, g_{2}(s)\right)\right| d s\right)^{p} d t+
$$$$
+\frac{2^{p} \mu^{p}}{(\gamma+\mu)^{p}(\Gamma(\alpha))^{p}} \int_{a}^{b}\left(\int_{a}^{b}(b-s)^{\alpha-1}\left|f\left(s, g_{1}(s)\right)-f\left(s, g_{2}(s)\right)\right| d s\right)^{p} d t
$$

Since $f(t, y)$ satisfies lipschitz condition on $\mathrm{D}$ with respect to $\mathrm{y}$. Therefore from inequality (3.9), we get:

$$
\begin{gathered}
\left\|T\left(g_{1}(t)\right)-T\left(g_{2}(t)\right)\right\|_{p}^{p} \leq \frac{2^{p} M^{p}}{(\Gamma(\alpha))^{p}} \int_{a}^{b}\left(\int_{a}^{t}(t-s)^{\alpha-1}\left|g_{1}(s)-g_{2}(s)\right| d s\right)^{p} d t+ \\
+\frac{2^{p} M^{p} \mu^{p}}{(\gamma+\mu)^{p}(\Gamma(\alpha))^{p}} \int_{a}^{b}\left(\int_{a}^{b}(b-s)^{\alpha-1}\left|g_{1}(s)-g_{2}(s)\right| d s\right)^{p} d t
\end{gathered}
$$

Since $g_{1}, g_{2} \in L^{p}(a, b)$ and $L^{p} \quad$ is a linear space, then $\left|g_{1}-g_{2}\right| \in L^{p}(a, b)$, $(t-s)^{\alpha-1} \in L^{p}(a, b)$ and $\quad(b-s)^{\alpha-1} \in L^{p}(a, b)$ where $\frac{1}{p}+\frac{1}{q}=1$, by using HÖlder inequality, equation (3.10) becomes 


$$
\begin{aligned}
& \left\|T\left(g_{1}(t)\right)-T\left(g_{2}(t)\right)\right\|_{p}^{p} \leq \frac{2^{p} M^{p}}{(\Gamma(\alpha))^{p}} \int_{a}^{b}\left(\left(\int_{a}^{t}(t-s)^{q(\alpha-1)} d s\right)^{\frac{1}{q}}\left(\int_{a}^{t}\left|g_{1}-g_{2}\right|^{p} d s\right)^{\frac{1}{p}}\right)^{p} d t+ \\
& +2^{p} \frac{M^{p} \mu^{p}}{(\gamma+\mu)^{p}(\Gamma(\alpha))^{p}} \int_{a}^{b}\left(\left(\int_{a}^{b}(b-s)^{q(\alpha-1)} d s\right)^{\frac{1}{q}}\left(\int_{a}^{b}\left|g_{1}-g_{2}\right|^{p} d s\right)^{\frac{1}{p}}\right)^{p} d t \\
& \left\|T\left(g_{1}(t)\right)-T\left(g_{2}(t)\right)\right\|_{p}^{p} \leq \frac{2^{p} M^{p}}{(\Gamma(\alpha))^{p}} \int_{a}^{b}\left(\frac{(t-a)^{p \alpha-1}}{\left(\frac{p \alpha-1}{p-1}\right)^{p-1}} \int_{a}^{t}\left|g_{1}-g_{2}\right|^{p} d s\right) d t+ \\
& +2^{p} \frac{M^{p} \mu^{p}}{(\gamma+\mu)^{p}(\Gamma(\alpha))^{p}} \int_{a}^{b}\left(\frac{(b-a)^{p \alpha-1}}{\left(\frac{p \alpha-1}{p-1}\right)^{p-1}} \int_{a}^{b}\left|g_{1}-g_{2}\right|^{p} d s\right) d t
\end{aligned}
$$

To integrate the right hand side of inequality (3.11), let:

$$
\begin{aligned}
& r(t)=\int_{a}^{t}\left|g_{1}-g_{2}\right|^{p} d s \\
& \left\|T\left(g_{1}(t)\right)-T\left(g_{2}(t)\right)\right\|_{p}^{p} \leq \frac{2^{p} M^{p}}{(\Gamma(\alpha))^{p}}\left(\frac{p-1}{p \alpha-1}\right)^{p-1} \int_{a}^{b}(t-a)^{p \alpha-1} r(t) d t+ \\
& +2^{p} \frac{M^{p} \mu^{p}}{(\gamma+\mu)^{p}(\Gamma(\alpha))^{p}}\left(\frac{p-1}{p \alpha-1}\right)^{p-1} \int_{a}^{b}\left((b-a)^{p \alpha-1} \int_{a}^{b}\left|g_{1}-g_{2}\right|^{p} d s\right) d t
\end{aligned}
$$

Integrate by parts, we have:

$$
\begin{aligned}
& d v=(t-a)^{p \alpha-1} \quad v=\frac{(t-a)^{p \alpha}}{p \alpha} \\
& u=r(t) \quad d u=r^{\prime}(t) d t \\
& \left\|T\left(g_{1}(t)\right)-T\left(g_{2}(t)\right)\right\|_{p}^{p} \leq \frac{2^{p} M^{p}}{(\Gamma(\alpha))^{p}}\left(\frac{p-1}{p \alpha-1}\right)^{p-1}\left[\frac{(b-a)^{p \alpha}}{p \alpha} \int_{a}^{b}\left|g_{1}-g_{1}\right|^{p} d t-\int_{a}^{b} \frac{(t-a)^{p \alpha}}{p \alpha} r^{\prime}(t) d t\right]+ \\
& +2^{p} \frac{M^{p} \mu^{p}}{(\gamma+\mu)^{p}(\Gamma(\alpha))^{p}}\left(\frac{p-1}{p \alpha-1}\right)^{p-1}(b-a)^{p \alpha-1} \int_{a}^{b}\left(\int_{a}^{b}\left|g_{1}-g_{2}\right|^{p} d s\right) d t
\end{aligned}
$$

Since $\int_{a}^{b} \frac{(t-a)^{p \alpha}}{p \alpha} r_{1}^{\prime}(t) d t \geq 0$, then inequality (3.12) gives

$\left\|T\left(g_{1}(t)\right)-T\left(g_{2}(t)\right)\right\|_{p}^{p} \leq \frac{2^{p} M^{p}}{(\Gamma(\alpha))^{p}}\left(\frac{p-1}{p \alpha-1}\right)^{p-1} \frac{(b-a)^{p \alpha}}{p \alpha}\left\|g_{1}-g_{2}\right\|_{p}^{p}+$ 


$$
\begin{gathered}
+2^{p} \frac{M^{p} \mu^{p}}{(\gamma+\mu)^{p}(\Gamma(\alpha))^{p}}\left(\frac{p-1}{p \alpha-1}\right)^{p-1}(b-a)^{p \alpha}\left\|g_{1}-g_{2}\right\|_{p}^{p} \\
\left\|T\left(g_{1}(t)\right)-T\left(g_{2}(t)\right)\right\|_{p} \leq \frac{2 M}{\Gamma(\alpha)}\left[\left(\frac{p-1}{p \alpha-1}\right)^{p-1}(b-a)^{p \alpha}\left(\frac{1}{p \alpha}+\frac{\mu^{p}}{(\gamma+\mu)^{p}}\right)\right]^{\frac{1}{p}}\left\|g_{1}-g_{2}\right\|_{p} \\
\text { where } \lambda=\frac{2 M(b-a)^{\alpha}}{\Gamma(\alpha)}\left(\frac{p-1}{p \alpha-1}\right)^{\frac{p-1}{p}} \sqrt[p]{\frac{1}{p \alpha}+\frac{\mu^{p}}{(\gamma+\mu)^{p}}}
\end{gathered}
$$

Thus $\mathrm{T}$ is contraction mapping and hence it has one and only one fixed point, $\operatorname{Ty}(\mathrm{t})=\mathrm{y}(\mathrm{t})$.

Example 3.3. Consider the following fractional differential equation:

$$
D^{0.7} y(t)=\frac{e^{2 t} y^{2}}{55.5\left(1+t^{2}\right)}
$$

with boundary condition

$$
y(0)+y(1)=1
$$

Here,

$$
\left|f\left(t, y_{1}\right)-f\left(t, y_{2}\right)\right|=\left|\frac{e^{2 t} y_{1}^{2}}{55.5\left(1+t^{2}\right)}-\frac{e^{2 t} y_{2}^{2}}{55.5\left(1+t^{2}\right)}\right| \leq \frac{1}{15}\left|y_{1}-y_{2}\right| \quad \forall\left(t, y_{1}\right),\left(t, y_{2}\right) \in D
$$

where $\gamma=1, \mu=1, p=2$ and the space is $\mathrm{L}^{2}(0,1)$, Thus

$$
\begin{aligned}
& \frac{2 M(b-a)^{\alpha}}{\Gamma(\alpha)}\left(\frac{p-1}{p \alpha-1}\right)^{\frac{p-1}{p}} \sqrt[p]{\frac{1}{p \alpha}+\frac{\mu^{p}}{(\gamma+\mu)^{p}}}<1 \Leftrightarrow \\
& (0.102717)(1.58113883)(0.9819805)=0.159483294<1 .
\end{aligned}
$$

Then by Theorem 3.2 , the function

$$
y(t)=\frac{1}{\Gamma(\alpha)} \int_{0}^{t}(t-s)^{-0.3} \frac{e^{2 s} y(s)^{2}}{55.5\left(1+s^{2}\right)} d s-\frac{1}{2 \Gamma(\alpha)} \int_{0}^{1}(1-s)^{-0.3} \frac{e^{2 s} y(s)^{2}}{55.5\left(1+s^{2}\right)} d s+\frac{1}{2}
$$

Is the only solution for the given fractional differential equation which satisfies the given boundary condition. 


\section{$\underline{\text { REFERENCES }}$}

[1] H.L. Arora and J.G. Al-Shamani, Stability of differential equation of non-integer order through fixed point in the large, Indian J. pure, appl. Math., 3 (1980), 307313.

[2] J.H. Barrett, Differential equations of noninteger order, Canad. J. Math., 6 (1954), 529-541.

[3] A.M. Bassam, Some existence theorems on differential equations of generalized order (presented to mathematical association of America-Texas section), April, 10(1964).

[4] M. Benchohra, S. Hamani and S.K. Ntouyas, Boundary value problems for differential equations with fractional order. Surveys Mathematics and its Applications Volume 3(2008), 1- 12.

[5] S.B. Hadid, Local and global existence theorems on differential equations of non-int eger order, J. Fract. Calc., 7 (1995), 101-105.

[6] A. A. Kilbas, Hari M. Srivastava, and Juan J. Trujillo, Theory and Applications of Fractional differential equations. North-Holland Mathematics Studies, 204. Elsevier Science B.V., Amsterdam, (2006).

[7] S.K. Miller and B. Ross, An Introduction to the Fractional calculus and Fractional differential equation, A Wiley-Interscience publication, John Wiley \&Sons, New York, USA., (1993).

[8] S.M. Momani, Local and global uniqueness theorems on differential equations of non-integer order via Bihari's and Gronwall's inequalities, Revista Tecnica J., 23(1) (2000), 66-69.

[9] H.L. Royden, Real Analysis, Prentice-Hall of India Private Limited, New Delhi110001, (2005). 